Available online at http://jnfa.mathres.org
MATHES Journal of Nonlinear Functional Analysis
Ants

\title{
EXISTENCE RESULTS OF NONLOCAL BOUNDARY VALUE PROBLEMS FOR NONLINEAR FRACTIONAL $q$-INTEGRODIFFERENCE EQUATIONS
}

\author{
UMAPHON SRIPHANOMWAN, JESSADA TARIBOON, NICHAPHAT PATANARAPEELERT, THANIN \\ SITTHIWIRATTHAM*
}

\begin{abstract}
Nonlinear Dynamic Analysis Research Center,
Department of Mathematics, Faculty of Applied Science,

King Mongkut's University of Technology North Bangkok, Bangkok, Thailand
\end{abstract}

\begin{abstract}
In this paper, we study the existence and uniqueness of solutions to nonlinear fractional $q$-integrodifference equations with nonlocal boundary value conditions. The governing problem consists of two different fractional orders and five different numbers of $q$ in derivatives and integrals. The Banach's contraction mapping principle and Krasnoselskii's fixed point theorem are employed to achieve the main results. In addition, an example illustrating our results is provided.
\end{abstract}

Keywords. Existence; $q$-integrodifference equation; Nonlocal boundary value problem; Fixed point theorem.

2010 Mathematics Subject Classification. 34A08, 34B18, 39A13.

\section{Introduction}

Fractional calculus is a generalization of the integer order of ordinary differentiation and integration to arbitrary order [1,2]. Recently, several reserchs in fractional calculus were published in engineering, economic, physical, and biological fields [3]. Especially, advances of

${ }^{*}$ Corresponding author.

E-mail addresses: u.sriphanomwan@gmail.com (U. Sriphanomwan), jessada.t@ sci.kmutnb.ac.th (J. Tariboon), nichaphat.p@ @sci.kmutnb.ac.th (N. Patanarapeelert), thanin.s@ sci.kmutnb.ac.th (T. Sitthiwirattham).

Received December 22, 2016; Accepted April 11, 2017.

(C)2017 Journal of Nonlinear Functional Analysis 
fractional calculus are dominated by applications in boundary value problems for fractional differential equations $[4,5,6]$. The discrete fractional calculus is a relatively new mathematical field. Therefore, many researchers have attempted to develop the theory of discrete fractional calculus in various directions. For some recent results, see [7] and the references therein. In medical science, Atici and Sengl have shown the usefulness of fractional difference equations in tumor growth modeling; see [8] and the references therein.

Usually, the exact solutions of the nonlinear equations are very difficult to obtain. Although we can use numerical approach to find the approximation of solution, researchers should ensure that the real problem has a solution to prevent the loss of time from finding a solution that does not exists. Based on this idea, both time and economic value will be saved. Recently, many researchers pay attention to investigate sufficient condtions to grarantee that the problem has solutions. The following works are the approaches of the study of the boundary value problems for fractional $q$-difference equations.

In 2013, Yang [9] proposed the boundary value problem of the fractional $q$-difference equation with $\phi$-Laplacian operator

$$
\left\{\begin{array}{l}
D_{q}^{\beta}\left(\phi_{\mu}\left(D_{q}^{\alpha} u(t)\right)\right)=f(t, u(t)), 0<t<1, \\
u(0)=u(1)=0, \quad D_{q}^{\alpha} u(0)=D_{q}^{\alpha} u(1)=0,
\end{array}\right.
$$

where $0<q<1,1<\alpha, \beta \leq 2$ and $D_{q}^{\alpha}$ is the $\alpha$-order fractional $q$-derivative of the RiemannLiouville type, $f:[0, T] \times \mathbb{R} \rightarrow \mathbb{R}$ is a continuous function, $\phi_{\mu}(s)=|s|^{\mu-2} s, \mu>1$ and $\left(\phi_{\mu}\right)^{-1}=$ $\phi_{v}, \frac{1}{\mu}+\frac{1}{v}=1$.

Zhao, Chen and Zhang [10] studied the fractional $q$-difference equation with nonlocal $q$ integral boundary conditions

$$
\left\{\begin{array}{l}
\left(D_{q}^{\alpha} u\right)(t)+f(t, u(t))=0,0<t<1 \\
u(0)=0, \quad u(1)=\mu I_{q}^{\beta} u(\eta)=\mu \int_{0}^{\eta} \frac{(\eta-q s)^{(\beta-1)}}{\Gamma_{q}(\beta)} u(s) d_{q} s
\end{array}\right.
$$

where $0<q<1,1<\alpha, \beta \leq 2,0<\eta<1, \mu>0, D_{q}^{\alpha}$ is the $\alpha$-order fractional $q$-derivative of the Riemann-Liouville type and $f:[0, T] \times \mathbb{R} \rightarrow \mathbb{R}$ is a continuous function. 
In 2014, Pongarm, Asawasamrit, Tariboon and Ntouyas [11] investigated the following fractional $q$-difference equation for multi-strip fractional $q$-integral boundary conditions

$$
\left\{\begin{array}{l}
D_{q}^{\alpha} u(t)=f(t, u(t)), t \in(0, T) \\
u(0)=0 \\
u(T)=\left.\sum_{i=1}^{m} \gamma_{i}\left(I_{q_{i}}^{\beta_{i}} u\right)\right|_{\eta_{i}} ^{\xi_{i}}=\sum_{i=1}^{m} \gamma_{i}\left(I_{q_{i}}^{\beta_{i}} u\left(\xi_{i}\right)-I_{q_{i}}^{\beta_{i}} u\left(\eta_{i}\right)\right)
\end{array}\right.
$$

where $1<\alpha \leq 2,0<q, q_{i}<1, \beta_{i}>0,0 \leq \eta_{i}<\xi_{i} \leq T, \gamma_{i} \in \mathbb{R}$ for all $i=1,2, \ldots, m, D_{q}^{\alpha}$ is the $\alpha$-order fractional $q$-derivative of the Riemann-Liouville type, $I_{q_{i}}^{\beta_{i}}$ is the fractional $q_{i}$-integral of order $\beta_{i}$ and $f:[0, T] \times \mathbb{R} \rightarrow \mathbb{R}$ is a continuous function.

Recently, Sitthiwirattham [12] studied the existence of solutions for the following nonlinear fractional $q$-difference equation with nonlocal three-point fractional quantum-integral boundary conditions

$$
\left\{\begin{array}{l}
D_{q}^{\alpha} x(t)=f\left(t, x(t), D_{\omega}^{v} x(t)\right), t \in[0, T], \\
x(\eta)=\rho(x), \\
I_{p}^{\beta} g(T) x(T)=\frac{1}{\Gamma_{p}(\beta)} \int_{0}^{T} g(s)(T-p s)^{(\beta-1)} x(s) d_{p} s=0 .
\end{array}\right.
$$

In addition, the authors proposed the following nonlinear fractional $q$-integrodifference equation with the same boundary conditions

$$
D_{q}^{\alpha} x(t)=f\left(t, x(t), \Psi_{\omega}^{\gamma} x(t)\right), t \in[0, T]
$$

where $p, q, \omega \in(0,1), \alpha \in(1,2], v \in(0,1], \beta, \gamma>0$ and $\eta \in(0, T)$ are given constants, $D_{q}^{\alpha}, D_{\omega}^{v}$ are the Riemann-Liouville fractional $q$-derivative of order $\alpha$ and $\omega$-derivative of order $v$, respectively, $f \in C([0, T] \times \mathbb{R} \times \mathbb{R}, \mathbb{R})$ and $g \in C\left([0, T], \mathbb{R}^{+}\right)$are given functions, $\rho: C([0, T], \mathbb{R}) \rightarrow \mathbb{R}$ is a given functional.

Recently, Patanarapeelert, Sriphanomwan and Sitthiwirattham [13] considered a sequential $q$-integrodifference boundary value problem involving two different orders and six different 
numbers of $q$ in derivatives and integrals of the form

$$
\left\{\begin{array}{l}
D_{q}\left[\rho(t) D_{p}^{\gamma}\left(\kappa+D_{o}\right)\right] x(t)=f\left(t, x(t), D_{w}\left[e_{o}^{\kappa t} x(t)\right], \Psi_{v} x(t)\right), \\
x(0)=x(T) \\
\left(D_{o}\left[e_{o}^{\kappa t} x(t)\right]\right)_{t=0}=D_{o}\left[e_{o}^{\kappa T} x(T)\right] \\
I_{r}^{\theta} \sigma(t) x(T)=0
\end{array}\right.
$$

where $t \in I_{\alpha}^{T}:=\left\{\alpha^{k} T: k \in \mathbb{N}\right\} \cup\{0, T\}, \gamma, \theta \in(0,1], p=\frac{p_{1}}{p_{2}}, q=\frac{q_{1}}{q_{2}}, o=\frac{o_{1}}{o_{2}}, r=\frac{r_{1}}{r_{2}}, w=\frac{w_{1}}{w_{2}}, v=$ $\frac{v_{1}}{v_{2}}$, and $\alpha=\frac{1}{L C M\left(p_{2}, q_{2}, o_{2}, r_{2}, w_{2}, v_{2}\right)}$ are proper fractions with $w \leq o, L C M$ is the lest common multiple, $\kappa \leq \frac{1}{T}, \rho, \sigma \in C\left(I_{\alpha}^{T}, \mathbb{R}^{+}\right)$and $f \in C\left(I_{\alpha}^{T} \times \mathbb{R} \times \mathbb{R} \times \mathbb{R}, \mathbb{R}\right)$ are given functions.

Motivated by the above mentioned works, the following boundary value problem of the fractional $q$ - integrodifference equation involving different numbers of $q$ are considered in this paper.

$$
\left\{\begin{array}{l}
D_{q}^{\alpha}\left(D_{p}^{\beta}(1+\rho(t))\right) x(t)=f\left(t, x(t), D_{\theta}^{\mu} x(t), \Psi_{\omega}^{\nu} x(t)\right), \\
x(0)=x(\eta), \quad I_{r}^{\gamma} x(T)=\int_{0}^{T} \frac{(T-r s)^{(\gamma-1)}}{\Gamma_{r}(\gamma)} x(s) d_{r} s=g(x),
\end{array}\right.
$$

where $t \in I_{\chi}^{T}:=\left\{\chi^{k} T: k \in \mathbb{N}\right\} \cup\{0, T\}, 0<\alpha, \beta, \mu \leq 1,1<\alpha+\beta \leq 2, v, \gamma>0, \eta \in I_{\chi}^{T}-$ $\{0, T\}, p=\frac{p_{1}}{p_{2}}, q=\frac{q_{1}}{q_{2}}, r=\frac{r_{1}}{r_{2}}, \theta=\frac{\theta_{1}}{\theta_{2}}, \omega=\frac{\omega_{1}}{\omega_{2}}$ are simplest form of proper fractions and $\chi=$ $\frac{1}{L C M\left(p_{2}, q_{2}, r_{2}, \theta_{2}, \omega_{2}\right)}, L C M$ is lest common multiple, $\rho(t) \in C\left(I_{\chi}^{T}, \mathbb{R}^{+}\right), f \in C\left(I_{\chi}^{T} \times \mathbb{R} \times \mathbb{R} \times \mathbb{R}, \mathbb{R}\right)$ are given function, $g: C\left(I_{\chi}^{T}, \mathbb{R}\right) \rightarrow \mathbb{R}$ is given functional, and for $\varphi \in C\left(I_{\chi}^{T} \times I_{\chi}^{T},[0, \infty)\right)$

$$
\Psi_{\omega}^{v} x(t):=\left(I_{\omega}^{v} \varphi x\right)(t)=\frac{1}{\Gamma_{\omega}(v)} \int_{0}^{t}(t-\omega s)^{(v-1)} \varphi(t, s) x(s) d_{\omega} s .
$$

The paper is organized as follows. In Section 2, we recall some definitions and basic lemmas. In Section 3 and Section 4, we prove the existence and uniqueness results for the boundary value problem by employing Banach's contraction mapping principle and Krasnoselskii's fixed point theorem. Finally, an illustrative example is presented in the last section.

\section{Preliminaries}

We first introduce notations, definitions, and lemmas which are used in the main results. Let $q \in(0,1)$ and define

$$
[a]_{q}:=\frac{1-q^{a}}{1-q}, \quad a \in \mathbb{R}
$$


The $q$-analogue of the power function $(a-b)^{(n)}$ with $n \in \mathbb{N}_{0}:=[0,1,2, \ldots]$ is

$$
(a-b)^{(0)}:=1, \quad(a-b)^{(n)}:=\prod_{k=0}^{n-1}\left(a-b q^{k}\right), \quad a, b \in \mathbb{R} .
$$

More generally, if $\alpha \in \mathbb{R}$, then

$$
(a-b)^{(\alpha)}:=a^{\alpha} \prod_{n=0}^{\infty} \frac{1-\left(\frac{b}{a}\right) q^{n}}{1-\left(\frac{b}{a}\right) q^{\alpha+n}}, a \neq 0 .
$$

In particular, if $b=0$ then $a^{(\alpha)}=a^{\alpha}$. We also use the notation $0^{(\alpha)}=0$ for $\alpha>0$. The $q$-gamma function is defined by

$$
\Gamma_{q}(x):=\frac{(1-q)^{(x-1)}}{(1-q)^{x-1}}, \quad x \in \mathbb{R} \backslash\{0,-1,-2, \ldots\},
$$

and satisfies $\Gamma_{q}(x+1)=[x]_{q} \Gamma_{q}(x)$.

Remark 2.1. [14] If $\alpha>0$ and $a \leq b \leq t$, then $(t-a)^{(\alpha)} \geq(t-b)^{(\alpha)}$.

Definition 2.2. [15] For $\alpha \geq 0$ and $f$ defined on [0,T], the fractional $q$-integral of the RiemannLiouville type is defined by

$$
\begin{aligned}
\left(I_{q}^{\alpha} f\right)(x) & :=\frac{1}{\Gamma_{q}(\alpha)} \int_{0}^{x}(x-q t)^{(\alpha-1)} f(t) d_{q} t \\
& =\frac{x(1-q)}{\Gamma_{q}(\alpha)} \sum_{n=0}^{\infty} q^{n}\left(x-x^{n+1}\right)^{(\alpha-1)} f\left(x q^{n}\right) \\
& =\frac{x^{\alpha}(1-q)}{\Gamma_{q}(\alpha)} \sum_{n=0}^{\infty} q^{n}\left(1-q^{n+1}\right)^{(\alpha-1)} f\left(x q^{n}\right)
\end{aligned}
$$

and $\left(I_{q}^{0} f\right)(x)=f(x)$.

Definition 2.3. [16] For $\alpha \geq 0$ and $f$ defined on $[0, T]$, the fractional q-derivative of the Riemann-Liouville type of order $\alpha$ is defined by

$$
\left(D_{q}^{\alpha} f\right)(x):=\left(D_{q}^{m} I_{q}^{m-\alpha} f\right)(x), \alpha>0,
$$

and $\left(D_{q}^{0} f\right)(x)=f(x)$, where $m$ is the smallest integer that is greater than or equal to $\alpha$.

Definition 2.4. [17] For any $x, s>0$,

$$
\begin{aligned}
B_{q}(x, s) & :=\int_{0}^{1} t^{(x-1)}(1-q t)^{(s-1)} d_{q} t \\
& =(1-q) \sum_{n=0}^{\infty} q^{n}\left(1-q^{n+1}\right)^{(s-1)}\left(q^{n}\right)^{(x-1)}=\frac{\Gamma_{q}(x) \Gamma_{q}(s)}{\Gamma_{q}(x+s)},
\end{aligned}
$$

is called the $q$-bata function. 
Lemma 2.5. [15] Let $\alpha, \beta \geq 0$ and $f$ be a function defined on $[0, T]$. Then, the following formulas hold:

$$
\begin{aligned}
& \text { (i) }\left(I_{q}^{\beta} I_{q}^{\alpha} f\right)(x)=\left(I_{q}^{\alpha+\beta} f\right)(x), \\
& (i i)\left(D_{q}^{\alpha} I_{q}^{\alpha} f\right)(x)=f(x)
\end{aligned}
$$

Lemma 2.6. [16] Let $\alpha>0$ and $N$ be a positive integer. Then, the following equality holds:

$$
\left(I_{q}^{\alpha} D_{q}^{N} f\right)(x)=\left(D_{q}^{N} I_{q}^{\alpha} f\right)(x)-\sum_{k=0}^{N-1} \frac{x^{\alpha-N+k}}{\Gamma_{q}(\alpha+k-N+1)}\left(D_{q}^{k} f\right)(0) .
$$

Lemma 2.7. [11] Let $\alpha, \beta, \gamma \geq 0$ and $0<p, q, r<1$. Then, the following formulas hold:

$$
\begin{aligned}
& \text { (i) } I_{q}^{\alpha} \eta^{\beta}=\frac{\Gamma_{q}(\beta+1)}{\Gamma_{q}(\alpha+\beta+1)} \eta^{\alpha+\beta} \\
& \text { (ii) } I_{p}^{\beta} I_{q}^{\alpha}(\eta)=\frac{\Gamma_{p}(\alpha+1)}{\Gamma_{p}(\alpha+\beta+1) \Gamma_{q}(\alpha+1)} \eta^{\alpha+\beta} \\
& \text { (iii) } I_{r}^{\gamma} I_{p}^{\beta} I_{q}^{\alpha}(\eta)=\frac{\Gamma_{p}(\alpha+1) \Gamma_{r}(\alpha+\beta+1)}{\Gamma_{p}(\alpha+\beta+1) \Gamma_{q}(\alpha+1) \Gamma_{r}(\alpha+\beta+\gamma+1)} \eta^{\alpha+\beta+\gamma}
\end{aligned}
$$

The following lemma which deals with a linear variant of the boundary value problems (1.7) is introduced to define the solution of this problem.

Lemma 2.8. Let $0<\alpha, \beta \leq 1,1<\alpha+\beta \leq 2, \gamma>0, \eta \in I_{\phi}^{T}-\{0, T\}, p=\frac{p_{1}}{p_{2}}, q=\frac{q_{1}}{q_{2}}, r=\frac{r_{1}}{r_{2}}$ be simplest proper fractions, $\phi=\frac{1}{L C M\left(p_{2}, q_{2}, r_{2}\right)}$, functions $y(t) \in C\left(I_{\phi}^{T}, \mathbb{R}\right)$ and $\rho(t) \in C\left(I_{\phi}^{T}, \mathbb{R}^{+}\right)$, and a functional $g: C\left(I_{\phi}^{T}, \mathbb{R}\right) \rightarrow \mathbb{R}$. Then the boundary value problem

$$
\begin{gathered}
D_{q}^{\alpha}\left(D_{p}^{\beta}(1+\rho(t))\right) x(t)=y(t), t \in I_{\phi}^{T}, \\
x(0)=x(\eta), \quad I_{r}^{\gamma} x(T)=g(x),
\end{gathered}
$$

is equivalent to the integral equation

$$
\begin{aligned}
x(t) & =(1+\rho(t))^{-1} I_{p}^{\beta} I_{q}^{\alpha} y(t)+\left[( \eta t ) ^ { \beta - 1 } ( t ^ { \alpha } - \eta ^ { \alpha } ) \left(g(x)-I_{r}^{\gamma}\left((1+\rho(s))^{-1}\right.\right.\right. \\
& \left.\left.\left.\times I_{p}^{\beta} I_{q}^{\alpha} y\right)(T)\right)+t^{\beta-1} I_{p}^{\beta} I_{q}^{\alpha} y(\eta)\left(t^{\alpha} I_{r}^{\gamma} \Omega_{2}(T)-I_{r}^{\gamma} \Omega_{1}(T)\right)\right] / \\
& {\left[(1+\rho(t)) \eta^{\beta-1}\left(I_{r}^{\gamma} \Omega_{1}(T)-\eta^{\alpha} I_{r}^{\gamma} \Omega_{2}(T)\right)\right] }
\end{aligned}
$$

where $\Omega_{1}(s)=\frac{s^{\alpha+\beta-1}}{1+\rho(s)}, \Omega_{2}(s)=\frac{s^{\beta-1}}{1+\rho(s)}, s \in I_{\phi}^{T}$, and $I_{r}^{\gamma} \Omega_{1}(T)-\eta^{\alpha} I_{r}^{\gamma} \Omega_{2}(T) \neq 0$. 
Proof. By Definition 2.3 and Lemma 2.5, we obtain

$$
I_{q}^{\alpha} D_{q}^{1} I_{q}^{1-\alpha}\left(D_{p}^{\beta}(1+\rho(t))\right) x(t)=I_{q}^{\alpha} y(t)
$$

which leads to

$$
D_{p}^{\beta}(1+\rho(t)) x(t)=I_{q}^{\alpha} y(t)+C_{1} t^{\alpha-1}
$$

and

$$
x(t)=\frac{1}{1+\rho(t)}\left(I_{p}^{\beta} I_{q}^{\alpha} y(t)+C_{1} \frac{\Gamma_{p}(\alpha)}{\Gamma_{p}(\alpha+\beta)} t^{\alpha+\beta-1}+C_{2} t^{\beta-1}\right) .
$$

From the first condition of (2.2), we have

$$
C_{1} \frac{\Gamma_{p}(\alpha)}{\Gamma_{p}(\alpha+\beta)} \eta^{\alpha+\beta-1}+C_{2} \eta^{\beta-1}=-I_{p}^{\beta} I_{q}^{\alpha} y(\eta) .
$$

For some $g: C\left(I_{\phi}^{T}, \mathbb{R}\right) \rightarrow \mathbb{R}$, we assume that $g(x)=\xi$, where $\xi \in \mathbb{R}$. After taking fractional $r$-integral of order $\gamma>0$ for (2.6), and employing the second condition of (2.2), we get

$$
C_{1} \frac{\Gamma_{p}(\alpha)}{\Gamma_{p}(\alpha+\beta)} I_{r}^{\gamma} \Omega_{1}(T)+C_{2} I_{r}^{\gamma} \Omega_{2}(T)=\xi-I_{r}^{\gamma}\left(\frac{1}{1+\rho(s)} I_{p}^{\beta} I_{q}^{\alpha} y\right)(T) .
$$

Solving the system of linear equations (2.7) and (2.8), we have the unknown constants $C_{1}$ and $C_{2}$ as given by

$$
\begin{aligned}
C_{1}= & \frac{\Gamma_{p}(\alpha+\beta)\left(\xi \eta^{\beta-1}+I_{r}^{\gamma} \Omega_{2}(T) I_{p}^{\beta} I_{q}^{\alpha} y(\eta)-\eta^{\beta-1} I_{r}^{\gamma}\left((1+\rho(s))^{-1} I_{p}^{\beta} I_{q}^{\alpha} y\right)(T)\right)}{\Gamma_{p}(\alpha) \eta^{\beta-1}\left(I_{r}^{\gamma} \Omega_{1}(T)-\eta^{\alpha} I_{r}^{\gamma} \Omega_{2}(T)\right)} \\
C_{2}= & \frac{\eta^{\alpha+\beta-1} I_{r}^{\gamma}\left((1+\rho(s))^{-1} I_{p}^{\beta} I_{q}^{\alpha} y\right)(T)-\xi \eta^{\alpha+\beta-1}-I_{r}^{\gamma} \Omega_{1}(T) I_{p}^{\beta} I_{q}^{\alpha} y(\eta)}{\eta^{\beta-1}\left(I_{r}^{\gamma} \Omega_{1}(T)-\eta^{\alpha} I_{r}^{\gamma} \Omega_{2}(T)\right)} .
\end{aligned}
$$

Substituting the constants $C_{1}$ and $C_{2}$ into (2.6) and replacing $\xi$ by $g(x)$, we obtain (2.3).

On the other hand, we shall show that (2.3) is the solution of problem (2.1)-(2.2). First of all, for (2.3) can alternatively be written as

$$
\begin{aligned}
(1+\rho(t)) x(t) & =I_{p}^{\beta} I_{q}^{\alpha} y(t)+\left[\eta ^ { \beta - 1 } ( t ^ { \alpha + \beta - 1 } - \eta ^ { \alpha } t ^ { \beta - 1 } ) \left(g(x)-I_{r}^{\gamma}\left((1+\rho(s))^{-1}\right.\right.\right. \\
& \left.\left.\left.\times I_{p}^{\beta} I_{q}^{\alpha} y\right)(T)\right)+I_{p}^{\beta} I_{q}^{\alpha} y(\eta)\left(t^{\alpha+\beta-1} I_{r}^{\gamma} \Omega_{2}(T)-t^{\beta-1} I_{r}^{\gamma} \Omega_{1}(T)\right)\right] / \\
& {\left[\eta^{\beta-1}\left(I_{r}^{\gamma} \Omega_{1}(T)-\eta^{\alpha} I_{r}^{\gamma} \Omega_{2}(T)\right)\right] . }
\end{aligned}
$$


Taking the fractional $p$-derivative of order $\beta$ for (2.9), we have

$$
\begin{aligned}
D_{p}^{\beta}(1+\rho(t)) x(t) & =I_{q}^{\alpha} y(t)+\left[\frac { \Gamma _ { p } ( \alpha + \beta ) } { \Gamma _ { p } ( \alpha + 1 ) } \eta ^ { \beta - 1 } t ^ { \alpha - 1 } \left(g(x)-I_{r}^{\gamma}\left((1+\rho(s))^{-1}\right.\right.\right. \\
& \left.\left.\left.\times I_{p}^{\beta} I_{q}^{\alpha} y\right)(T)\right)+\frac{\Gamma_{p}(\alpha+\beta)}{\Gamma_{p}(\alpha+1)} t^{\alpha-1} I_{p}^{\beta} I_{q}^{\alpha} y(\eta) I_{r}^{\gamma} \Omega_{2}(T)\right] / \\
& {\left[\eta^{\beta-1}\left(I_{r}^{\gamma} \Omega_{1}(T)-\eta^{\alpha} I_{r}^{\gamma} \Omega_{2}(T)\right)\right] . }
\end{aligned}
$$

Finally, taking the fractional $q$-derivative of order $\alpha$ for (2.10), we obtain (2.1). This completes the proof.

\section{Main results}

Let $\mathscr{C}=C\left(I_{\chi}^{T}, \mathbb{R}\right)$ be a Banach space of all continuous functions from $I_{\chi}^{T}$ to $\mathbb{R}$. Define the norm by $\|x\|_{\mathscr{C}}=\max \left\{\|x\|,\left\|D_{\theta}^{\mu} x\right\|\right\}$, where $\|x\|=\sup _{t \in I_{\chi}^{T}}|x(t)|$ and $\left\|D_{\theta}^{\mu} x\right\|=\sup _{t \in I_{\chi}^{T}}\left|D_{\theta}^{\mu} x(t)\right|$. The operator $\mathscr{A}: \mathscr{C} \rightarrow \mathscr{C}$ is defined by

$$
\begin{aligned}
(\mathscr{A} x)(t) & :=(1+\rho(t))^{-1} I_{p}^{\beta} I_{q}^{\alpha} f\left(u, x, D_{\theta}^{\mu} x, \Psi_{\omega}^{\nu} x\right)(t)+\left[(\eta t)^{\beta-1}\left(t^{\alpha}-\eta^{\alpha}\right)\right. \\
& \times\left(g(x)-I_{r}^{\gamma}\left((1+\rho(s))^{-1} I_{p}^{\beta} I_{q}^{\alpha} f\left(v, x, D_{\theta}^{\mu} x, \Psi_{\omega}^{\nu} x\right)\right)(T)\right) \\
& \left.+t^{\beta-1} I_{p}^{\beta} I_{q}^{\alpha} f\left(u, x, D_{\theta}^{\mu} x, \Psi_{\omega}^{\nu} x\right)(\eta)\left(t^{\alpha} I_{r}^{\gamma} \Omega_{2}(T)-I_{r}^{\gamma} \Omega_{1}(T)\right)\right] / \\
& {\left[(1+\rho(t)) \eta^{\beta-1}\left(I_{r}^{\gamma} \Omega_{1}(T)-\eta^{\alpha} I_{r}^{\gamma} \Omega_{2}(T)\right)\right] . }
\end{aligned}
$$

Since problem (1.7) has solutions if and only if the operator $\mathscr{A}$ has fixed points, our first result is based on the Banach's fixed point theorem.

Theorem 3.1. Assume that the functional $g: C\left(I_{\chi}^{T}, \mathbb{R}\right) \rightarrow \mathbb{R}$ is continuous, $f: I_{\chi}^{T} \times \mathbb{R} \times \mathbb{R} \times$ $\mathbb{R} \rightarrow \mathbb{R}$ is continuous, $\rho: I_{\chi}^{T} \rightarrow \mathbb{R}$ and $\varphi: I_{\chi}^{T} \times I_{\chi}^{T} \rightarrow[0, \infty)$ are continuous functions. Let $\varphi_{0}=\sup _{(t, s) \in I_{\chi}^{T} \times I_{\chi}^{T}}\{\varphi(t, s)\}$. Suppose the following conditions $\left(H_{1}\right)$ there exist positive numbers $L_{1}, L_{2}, L_{3}$ such that, for each $t \in I_{\chi}^{T}$ and $x, y \in \mathscr{C}$,

$$
\left|f\left(t, x, D_{\theta}^{\mu} x, \Psi_{\omega}^{v} x\right)-f\left(t, y, D_{\theta}^{\mu} y, \Psi_{\omega}^{v} y\right)\right| \leq L_{1}\|x-y\|+L_{2}\left\|D_{\theta}^{\mu} x-D_{\theta}^{\mu} y\right\|+L_{3}\left\|\Psi_{\omega}^{v} x-\Psi_{\omega}^{v} y\right\| .
$$

$\left(H_{2}\right)$ There exists a positive number $\tau$ such that, for each $x, y \in \mathscr{C},|g(x)-g(y)| \leq \tau\|x-y\|$. $\left(H_{3}\right)$ For each $t \in I_{\chi}^{T}, 0<n<\rho(t)<N$. 
$\left(H_{4}\right) \Theta:=\lambda\left(P_{1}+P_{2}\right)+\frac{\Gamma_{q}(\alpha+1) \Gamma_{p}(\alpha+\beta+1) \Gamma_{r}(\alpha+\beta+\gamma+1)(1+n)}{\Gamma_{p}(\alpha+1) \Gamma_{r}(\alpha+\beta+1) T^{\alpha+\beta+\gamma}} \tau P_{2}<1$,

where

$$
\begin{aligned}
\lambda & =\max \left\{L_{1}+L_{2}+L_{3} \frac{\varphi_{0} T^{v}}{\Gamma_{\omega}(v+1)}\right\}, \\
\Lambda_{1} & =\left|\Gamma_{r}(\beta) \Gamma_{r}(\alpha+\beta+\gamma)-\Gamma_{r}(\alpha+\beta) \Gamma_{r}(\beta+\gamma)\right|, \\
\Lambda_{2} & =\left|\Gamma_{r}(\alpha+\beta) \Gamma_{r}(\beta+\gamma) T^{\alpha}-\Gamma_{r}(\beta) \Gamma_{r}(\alpha+\beta+\gamma) \eta^{\alpha}\right| \neq 0, \\
P_{1} & =\frac{\Gamma_{p}(\alpha+1) T^{\alpha+\beta}}{\Gamma_{q}(\alpha+1) \Gamma_{p}(\alpha+\beta+1)(1+n)}+\frac{\Gamma_{p}(\alpha+1)(1+N) \eta^{\alpha+1} T^{\alpha+\beta-1} \Lambda_{1}}{\Gamma_{q}(\alpha+1) \Gamma_{p}(\alpha+\beta+1)(1+n)^{2} \Lambda_{2}}, \\
P_{2} & =\frac{\Gamma_{p}(\alpha+1) \Gamma_{r}(\beta+\gamma) \Gamma_{r}(\alpha+\beta+1) \Gamma_{r}(\alpha+\beta+\gamma)(1+N)\left(T^{\alpha}-\eta^{\alpha}\right) T^{\alpha+\beta}}{\Gamma_{q}(\alpha+1) \Gamma_{p}(\alpha+\beta+1) \Gamma_{r}(\alpha+\beta+\gamma+1)(1+n)^{2} \Lambda_{2}} .
\end{aligned}
$$

Then the boundary value problem (1.7) has a unique solution.

Proof. We begin the proof by transforming the boundary value problem (1.7) into a fixed point problem $x=\mathscr{A} x$, where $\mathscr{A}: \mathscr{C} \rightarrow \mathscr{C}$ is defined by (3.1). Assuming that $\sup |f(t, 0,0,0)|=M$ and $\sup _{x \in \mathscr{C}}|g(x)|=K$, we choose a constant $R$ satisfied with

$$
R \geq \frac{M\left(P_{1}+P_{2}\right)+\frac{\Gamma_{q}(\alpha+1) \Gamma_{p}(\alpha+\beta+1) \Gamma_{r}(\alpha+\beta+\gamma+1)(1+n)}{\Gamma_{p}(\alpha+1) \Gamma_{r}(\alpha+\beta+1) T^{\alpha+\beta+\gamma}} K P_{2}}{1-\Theta} .
$$

We next show that $\mathscr{A} B_{R} \subset B_{R}$, where $B_{R}=\left\{x \in \mathscr{C}:\|x\|_{\mathscr{C}} \leq R\right\}$. For all $x, y \in \mathscr{C}$ and for each $t \in I_{\chi}^{T}$, we have

$$
\begin{aligned}
|\mathscr{A} x| \leq & (1+\rho(t))^{-1} I_{p}^{\beta} I_{q}^{\alpha}\left|f\left(u, x, D_{\theta}^{\mu} x, \Psi_{\omega}^{v} x\right)\right|(t)+\left[(\eta t)^{\beta-1}\left|t^{\alpha}-\eta^{\alpha}\right| \mid g(x)\right. \\
& -I_{r}^{\gamma}\left((1+\rho(s))^{-1} I_{p}^{\beta} I_{q}^{\alpha}\left|f\left(v, x, D_{\theta}^{\mu} x, \Psi_{\omega}^{v} x\right)\right|\right)(T)\left|+t^{\beta-1} I_{p}^{\beta} I_{q}^{\alpha}\right| f\left(u, x, D_{\theta}^{\mu} x, \Psi_{\omega}^{v} x\right) \mid(\eta) \\
& \left.\times\left|t^{\alpha} I_{r}^{\gamma} \Omega_{2}(T)-I_{r}^{\gamma} \Omega_{1}(T)\right|\right] /\left[(1+\rho(t)) \eta^{\beta-1}\left|I_{r}^{\gamma} \Omega_{1}(T)-\eta^{\alpha} I_{r}^{\gamma} \Omega_{2}(T)\right|\right] \\
\leq & (1+n)^{-1} I_{p}^{\beta} I_{q}^{\alpha}\left|f\left(u, x, D_{\theta}^{\mu} x, \Psi_{\omega}^{v} x\right)\right|(T)+\left[(\eta T)^{\beta-1}\left|T^{\alpha}-\eta^{\alpha}\right|(|g(x)|\right. \\
& \left.+(1+n)^{-1} I_{r}^{\gamma} I_{p}^{\beta} I_{q}^{\alpha}\left|f\left(v, x, D_{\theta}^{\mu} x, \Psi_{\omega}^{v} x\right)\right|(T)\right)+T^{\beta-1} I_{p}^{\beta} I_{q}^{\alpha}\left|f\left(u, x, D_{\theta}^{\mu} x, \Psi_{\omega}^{v} x\right)\right|(\eta) \\
& \left.\times\left|T^{\alpha} I_{r}^{\gamma} \Omega_{2}(T)-I_{r}^{\gamma} \Omega_{1}(T)\right|\right] /\left[(1+n) \eta^{\beta-1}\left|I_{r}^{\gamma} \Omega_{1}(T)-\eta^{\alpha} I_{r}^{\gamma} \Omega_{2}(T)\right|\right] .
\end{aligned}
$$


Applying the following inequalities

$$
\begin{aligned}
\left|f\left(t, x, D_{\theta}^{\mu} x, \Psi_{\omega}^{v} x\right)\right| & \leq\left|f\left(t, x, D_{\theta}^{\mu} x, \Psi_{\omega}^{v} x\right)-f(t, 0,0,0)\right|+|f(t, 0,0,0)| \\
& \leq L_{1}\|x\|+L_{2}\left\|D_{\theta}^{\mu} x\right\|+L_{3} \frac{\varphi_{0} T^{v}}{\Gamma_{\omega}(v+1)}\|x\|+M \\
& \leq \lambda R+M,
\end{aligned}
$$

and

$$
|g(x)| \leq|g(x)-g(0)|+|g(0)| \leq \tau R+K
$$

we have

$$
\begin{aligned}
&|\mathscr{A} x| \leq \frac{(\lambda R+M) \Gamma_{p}(\alpha+1) T^{\alpha+\beta}}{\Gamma_{q}(\alpha+1) \Gamma_{p}(\alpha+\beta+1)(1+n)}+\left[(\eta T)^{\beta-1}\left(T^{\alpha}-\eta^{\alpha}\right)((\tau R+K)\right. \\
&\left.+\frac{(\lambda R+M) \Gamma_{p}(\alpha+1) \Gamma_{r}(\alpha+\beta+1) T^{\alpha+\beta+\gamma}}{\Gamma_{q}(\alpha+1) \Gamma_{p}(\alpha+\beta+1) \Gamma_{r}(\alpha+\beta+\gamma+1)(1+n)}\right) \\
&\left.+\frac{(\lambda R+M) \Gamma_{p}(\alpha+1) \eta^{\alpha+\beta} T^{\alpha+2 \beta+\gamma-2}}{\Gamma_{q}(\alpha+1) \Gamma_{p}(\alpha+\beta+1)(1+n)}\left|\frac{\Gamma_{r}(\beta)}{\Gamma_{r}(\beta+\gamma)}-\frac{\Gamma_{r}(\alpha+\beta)}{\Gamma_{r}(\alpha+\beta+\gamma)}\right|\right] / \\
& {\left[\frac{\left.(1+n) \eta^{\beta-1} T^{\beta+\gamma-1}\left|\frac{\Gamma_{r}(\alpha+\beta) T^{\alpha}}{\Gamma_{r}(\alpha+\beta+\gamma)}-\frac{\Gamma_{r}(\beta) \eta^{\alpha}}{\Gamma_{r}(\beta+\gamma)}\right|\right]}{=}\right.} \\
& R \Theta+M\left(P_{1}+P_{2}\right)+\frac{\Gamma_{q}(\alpha+1) \Gamma_{p}(\alpha+\beta+1) \Gamma_{r}(\alpha+\beta+\gamma+1)(1+n)}{\Gamma_{p}(\alpha+1) \Gamma_{r}(\alpha+\beta+1) T^{\alpha+\beta+\gamma}} K P_{2},
\end{aligned}
$$

and

$$
\begin{aligned}
& \left|D_{\theta}^{\mu} \mathscr{A} x\right|=\left|D_{\theta} I_{\theta}^{1-\mu} \mathscr{A} x\right| \\
\leq & \frac{(\lambda R+M) \Gamma_{p}(\alpha+1) \Gamma_{\theta}(\alpha+\beta+1)}{\Gamma_{q}(\alpha+1) \Gamma_{p}(\alpha+\beta+1) \Gamma_{\theta}(\alpha+\beta+2-\mu)(1+n)} D_{\theta}\left(t^{\alpha+\beta+1-\mu}\right) \\
+ & {\left[\left|\frac{\Gamma_{\theta}(\alpha+\beta) \eta^{\beta-1}}{\Gamma_{\theta}(\alpha+\beta+1-\mu)(1+n)} D_{\theta}\left(t^{\alpha+\beta-\mu}\right)-\frac{\Gamma_{\theta}(\beta) \eta^{\alpha+\beta-1}}{\Gamma_{\theta}(\beta+1-\mu)(1+n)} D_{\theta}\left(t^{\beta-\mu}\right)\right|\right.} \\
& \times\left((\tau R+K)+\frac{(\lambda R+M) \Gamma_{p}(\alpha+1) \Gamma_{r}(\alpha+\beta+1) T^{\alpha+\beta+\gamma}}{\Gamma_{q}(\alpha+1) \Gamma_{p}(\alpha+\beta+1) \Gamma_{r}(\alpha+\beta+\gamma+1)(1+n)}\right) \\
& +\frac{(\lambda R+M) \Gamma_{p}(\alpha+1) \eta^{\alpha+\beta}}{\Gamma_{q}(\alpha+1) \Gamma_{p}(\alpha+\beta+1)} \mid \frac{\Gamma_{r}(\beta) \Gamma_{\theta}(\alpha+\beta) T^{\beta+\gamma-1}}{\Gamma_{r}(\beta+\gamma) \Gamma_{\theta}(\alpha+\beta+1-\mu)(1+n)^{2}} D_{\theta}\left(t^{\alpha+\beta-\mu}\right) \\
- & \left.\frac{\Gamma_{r}(\alpha+\beta) \Gamma_{\theta}(\beta) T^{\alpha+\beta+\gamma-1}}{\Gamma_{r}(\alpha+\beta+\gamma) \Gamma_{\theta}(\beta+1-\mu)(1+n)^{2}} \mid\right] /\left[\frac{\eta^{\beta-1} T^{\beta+\gamma-1}}{1+N} \mid \frac{\Gamma_{r}(\alpha+\beta) T^{\alpha}}{\Gamma_{r}(\alpha+\beta+\gamma)}\right. \\
- & \left.\frac{\Gamma_{r}(\beta) \eta^{\alpha}}{\Gamma_{r}(\beta+\gamma)} \mid\right] .
\end{aligned}
$$


Since $D_{\theta} t^{\rho}=\frac{t^{\rho}\left(1-\theta^{\rho}\right)}{t(1-\theta)}$, where

$$
\rho \in\{\alpha+\beta+1-\mu, \alpha+\beta-\mu, \beta-\mu\}
$$

we have

$$
\begin{aligned}
& \left|D_{\theta}^{\mu} \mathscr{A} x\right| \\
& \leq(\lambda R+M)\left\{\frac{\Gamma_{p}(\alpha+1) T^{\alpha+\beta}}{\Gamma_{q}(\alpha+1) \Gamma_{p}(\alpha+\beta+1)(1+n)} \cdot \frac{\Gamma_{\theta}(\alpha+\beta+1)}{\Gamma_{\theta}(\alpha+\beta+2-\mu) T^{\mu}}\right. \\
& \times\left|\frac{1-\theta^{\alpha+\beta+1-\mu}}{1-\theta}\right|+\frac{\Gamma_{p}(\alpha+1)(1+N) \eta^{\alpha+1} T^{\alpha+\beta-1}}{\Gamma_{q}(\alpha+1) \Gamma_{p}(\alpha+\beta+1)(1+n)^{2} \Lambda_{2}} \\
& \times\left|\frac{\Gamma_{r}(\beta) \Gamma_{\theta}(\alpha+\beta)}{\Gamma_{r}(\beta+\gamma) \Gamma_{\theta}(\alpha+\beta+1-\mu) T^{\mu}}\right| \frac{1-\theta^{\alpha+\beta-\mu}}{1-\theta} \mid-\frac{\Gamma_{r}(\alpha+\beta) \Gamma_{\theta}(\beta)}{\Gamma_{r}(\alpha+\beta+\gamma) \Gamma_{\theta}(\beta+1-\mu) T^{\mu}} \\
& \times\left|\frac{1-\theta^{\beta-\mu}}{1-\theta}\right| \mid+\frac{\Gamma_{p}(\alpha+1) \Gamma_{r}(\beta+\gamma) \Gamma_{r}(\alpha+\beta+1) \Gamma_{r}(\alpha+\beta+\gamma)(1+N) T^{\alpha+\beta}}{\Gamma_{q}(\alpha+1) \Gamma_{p}(\alpha+\beta+1) \Gamma_{r}(\alpha+\beta+\gamma+1)(1+n)^{2} \Lambda_{2}} \\
& \left.\times\left|\frac{\Gamma_{\theta}(\alpha+\beta) T^{\alpha}}{\Gamma_{\theta}(\alpha+\beta+1-\mu) T^{\mu}}\right| \frac{1-\theta^{\alpha+\beta-\mu}}{1-\theta}\left|-\frac{\Gamma_{\theta}(\beta) \eta^{\alpha}}{\Gamma_{\theta}(\beta+1-\mu) T^{\mu}}\right| \frac{1-\theta^{\beta-\mu}}{1-\theta}||\right\} \\
& +(\tau R+K)\left\{\frac{\Gamma_{r}(\beta+\gamma) \Gamma_{r}(\alpha+\beta+\gamma)(1+N)}{(1+n) T^{\gamma} \Lambda_{2}}\left|\frac{\Gamma_{\theta}(\alpha+\beta) T^{\alpha}}{\Gamma_{\theta}(\alpha+\beta+1-\mu) T^{\mu}}\right| \frac{1-\theta^{\alpha+\beta-\mu}}{1-\theta} \mid\right. \\
& \left.-\frac{\Gamma_{\theta}(\beta) \eta^{\alpha}}{\Gamma_{\theta}(\beta+1-\mu) T^{\mu}}\left|\frac{1-\theta^{\beta-\mu}}{1-\theta}\right| \mid\right\} \\
& \leq(\lambda R+M)\left\{\frac{\Gamma_{p}(\alpha+1) T^{\alpha+\beta}}{\Gamma_{q}(\alpha+1) \Gamma_{p}(\alpha+\beta+1)(1+n)}+\frac{\Gamma_{p}(\alpha+1)(1+N) \eta^{\alpha+1} T^{\alpha+\beta-1} \Lambda_{1}}{\Gamma_{q}(\alpha+1) \Gamma_{p}(\alpha+\beta+1)(1+n)^{2} \Lambda_{2}}\right. \\
& \left.+\frac{\Gamma_{p}(\alpha+1) \Gamma_{r}(\beta+\gamma) \Gamma_{r}(\alpha+\beta+1) \Gamma_{r}(\alpha+\beta+\gamma)(1+N)\left(T^{\alpha}-\eta^{\alpha}\right) T^{\alpha+\beta}}{\Gamma_{q}(\alpha+1) \Gamma_{p}(\alpha+\beta+1) \Gamma_{r}(\alpha+\beta+\gamma+1)(1+n)^{2} \Lambda_{2}}\right\} \\
& +(\tau R+K)\left\{\frac{\Gamma_{r}(\beta+\gamma) \Gamma_{r}(\alpha+\beta+\gamma)(1+N)\left(T^{\alpha}-\eta^{\alpha}\right)}{(1+n) T^{\gamma} \Lambda_{2}}\right\} \\
& <R \Theta+M\left(P_{1}+P_{2}\right) \\
& +\frac{\Gamma_{q}(\alpha+1) \Gamma_{p}(\alpha+\beta+1) \Gamma_{r}(\alpha+\beta+\gamma+1)(1+n)}{\Gamma_{p}(\alpha+1) \Gamma_{r}(\alpha+\beta+1) T^{\alpha+\beta+\gamma}} K P_{2} .
\end{aligned}
$$

Therefore, $\|\mathscr{A} x\|_{\mathscr{C}} \leq R$ and hence $\mathscr{A} B_{R} \subset B_{R}$. We next show that $\mathscr{A}$ is a contraction. Denote that

$$
\mathscr{S}\left[t, x, y, D_{\theta}^{\mu} x, D_{\theta}^{\mu} y, \Psi_{\omega}^{v} x, \Psi_{\omega}^{v} y\right]=\left|f\left(t, x, D_{\theta}^{\mu} x, \Psi_{\omega}^{v} x\right)-f\left(t, y, D_{\theta}^{\mu} y, \Psi_{\omega}^{v} y\right)\right|
$$


For all $x, y \in \mathscr{C}$ and for each $t \in I_{\chi}^{T}$, we have

$$
\begin{aligned}
& |\mathscr{A} x-\mathscr{A} y| \\
\leq & (1+\rho(t))^{-1} I_{p}^{\beta} I_{q}^{\alpha} \mathscr{S}\left[t, x, y, D_{\theta}^{\mu} x, D_{\theta}^{\mu} y, \Psi_{\omega}^{v} x, \Psi_{\omega}^{v} y\right](t)+\left[(\eta t)^{\beta-1}\left|t^{\alpha}-\eta^{\alpha}\right|\right. \\
& \times|| g(x)-g(y)\left|-I_{r}^{\gamma}\left((1+\rho(s))^{-1} I_{p}^{\beta} I_{q}^{\alpha} \mathscr{S}\left[t, x, y, D_{\theta}^{\mu} x, D_{\theta}^{\mu} y, \Psi_{\omega}^{v} x, \Psi_{\omega}^{v} y\right]\right)(T)\right| \\
& \left.+t^{\beta-1} I_{p}^{\beta} I_{q}^{\alpha} \mathscr{S}\left[t, x, y, D_{\theta}^{\mu} x, D_{\theta}^{\mu} y, \Psi_{\omega}^{v} x, \Psi_{\omega}^{v} y\right](\eta)\left|t^{\alpha} I_{r}^{\gamma} \Omega_{2}(T)-I_{r}^{\gamma} \Omega_{1}(T)\right|\right] / \\
= & {\left[(1+\rho(t)) \eta^{\beta-1}\left|I_{r}^{\gamma} \Omega_{1}(T)-\eta^{\alpha} I_{r}^{\gamma} \Omega_{2}(T)\right|\right] } \\
& {\left[x-y \|_{\mathscr{C}} \Theta,\right.}
\end{aligned}
$$

and

$$
\begin{aligned}
& \left|D_{\theta}^{\mu} \mathscr{A} x-D_{\theta}^{\mu} \mathscr{A} y\right|=\left|D_{\theta}\left(I_{\theta}^{1-\mu} \mathscr{A} x-I_{\theta}^{1-\mu} \mathscr{A} y\right)\right| \\
= & \mid D_{\theta}\left\{I_{\theta}^{1-\mu}\left((1+\rho(s))^{-1} I_{p}^{\beta} I_{q}^{\alpha} \mathscr{S}\left[t, x, y, D_{\theta}^{\mu} x, D_{\theta}^{\mu} y, \Psi_{\omega}^{v} x, \Psi_{\omega}^{v} y\right]\right)(t)\right. \\
& +\left[\left(\eta^{\beta-1} I_{\theta}^{1-\mu}\left((1+\rho(s))^{-1} t^{\alpha+\beta-1}\right)-\eta^{\alpha+\beta-1} I_{\theta}^{1-\mu}\left((1+\rho(s))^{-1} t^{\beta-1}\right)\right)\right. \\
& \times\left(|g(x)-g(y)|-I_{r}^{\gamma}\left((1+\rho(s))^{-1} I_{p}^{\beta} I_{q}^{\alpha} \mathscr{S}\left[t, x, y, D_{\theta}^{\mu} x, D_{\theta}^{\mu} y, \Psi_{\omega}^{v} x, \Psi_{\omega}^{v} y\right]\right)(T)\right) \\
& +I_{p}^{\beta} I_{q}^{\alpha} \mathscr{S}\left[t, x, y, D_{\theta}^{\mu} x, D_{\theta}^{\mu} y, \Psi_{\omega}^{v} x, \Psi_{\omega}^{v} y\right](\eta)\left(I_{r}^{\gamma} \Omega_{2}(T) I_{\theta}^{1-\mu}\left((1+\rho(s))^{-1} t^{\alpha+\beta-1}\right)\right. \\
& \left.\left.\left.-I_{r}^{\gamma} \Omega_{1}(T) I_{\theta}^{1-\mu}\left((1+\rho(s))^{-1} t^{\beta-1}\right)\right)\right] /\left[\eta^{\beta-1}\left(I_{r}^{\gamma} \Omega_{1}(T)-\eta^{\alpha} I_{r}^{\gamma} \Omega_{2}(T)\right)\right]\right\} \mid \\
\leq & \lambda\|x-y\|_{\mathscr{C}}\left\{\frac{\Gamma_{p}(\alpha+1) T^{\alpha+\beta}}{\Gamma_{q}(\alpha+1) \Gamma_{p}(\alpha+\beta+1)(1+n)}+\frac{\Gamma_{p}(\alpha+1)(1+N) \eta^{\alpha+1} T^{\alpha+\beta-1} \Lambda_{1}(\alpha+1) \Gamma_{p}(\alpha+\beta+1)(1+n)^{2} \Lambda_{2}}{\Gamma_{2}}\right. \\
& \left.+\frac{\Gamma_{p}(\alpha+1) \Gamma_{r}(\beta+\gamma) \Gamma_{r}(\alpha+\beta+1) \Gamma_{r}(\alpha+\beta+\gamma)(1+N)\left(T^{\alpha}-\eta^{\alpha}\right) T^{\alpha+\beta}}{\Gamma_{q}(\alpha+1) \Gamma_{p}(\alpha+\beta+1) \Gamma_{r}(\alpha+\beta+\gamma+1)(1+n)^{2} \Lambda_{2}}\right\} \\
& +\tau\|x-y\|_{\mathscr{C}}\left\{\frac{\Gamma_{r}(\beta+\gamma) \Gamma_{r}(\alpha+\beta+\gamma)(1+N)\left(T^{\alpha}-\eta^{\alpha}\right)}{(1+n) T_{\gamma}^{\gamma} \Lambda_{2}}\right\} \\
= & \|x-y\|_{\mathscr{C}} \Theta .
\end{aligned}
$$

Thus, $\|\mathscr{A} x-\mathscr{A} y\|_{\mathscr{C}} \leq \Theta\|x-y\|_{\mathscr{C}}$. From $\left(H_{4}\right)$, we can conclude that $\mathscr{A}$ is a contraction. Hence, the conclusion of the theorem is followed by Banach's contraction mapping principle. This completes the proof. 
The following Krasnoselskii's fixed point theorem is introduced to accomphished the second result.

Theorem 3.2. (Krasnoselskii fixed point theorem) [18] Let $K$ be a bounded closed convex and nonempty subset of a Banach space $X$. Let A,B be operators such that

(i) $A x+B y \in K$ whenever $x, y \in K$,

(ii) A is compact and continuous,

(iii) B is a contraction mapping.

Then there exists $z \in K$ such that $z=A z+B z$.

Theorem 3.3. (Arzela-Ascoli theorem) [18] Let $D \subseteq \mathbb{R}^{n}$ be a bounded domain, $K \subseteq C(\bar{D}, \mathbb{R})$ be bounded and the following property of equicontinuity holds. For every $\varepsilon>0$, there exists $\delta>0$, so that

$$
\|x-y\|<\delta \Rightarrow|u(x)-u(y)|<\varepsilon, \forall x, y \in \bar{D}, \forall u \in K
$$

Then $\bar{K}$ is compact.

Theorem 3.4. Assume that $\left(\mathrm{H}_{2}\right)-\left(\mathrm{H}_{3}\right)$ hold. In addition, $f: I_{\chi}^{T} \times \mathbb{R} \times \mathbb{R} \times \mathbb{R} \rightarrow \mathbb{R}$ is a continuous function satisfying the following condition:

$\left(H_{5}\right)$ For all $\left(t, x, D_{\theta}^{\mu} x, \Psi_{\omega}^{\nu}\right) \in I_{\chi}^{T} \times \mathbb{R} \times \mathbb{R} \times \mathbb{R}$, with $\mu \in C\left(I_{\chi}^{T}, \mathbb{R}^{+}\right),\left|f\left(t, x, D_{\theta}^{\mu} x, \Psi_{\omega}^{\nu}\right)\right| \leq \mu(t)$. If

$$
\Phi:=\|\mu\|\left(P_{1}+P_{2}\right)+\frac{\Gamma_{q}(\alpha+1) \Gamma_{p}(\alpha+\beta+1) \Gamma_{r}(\alpha+\beta+\gamma+1)(1+n)}{\Gamma_{p}(\alpha+1) \Gamma_{r}(\alpha+\beta+1) T^{\alpha+\beta+\gamma}} \tau P_{2}<1,
$$

then the boundary value problem (1.7) has at least one solution on $I_{\chi}^{T}$.

Proof. Let $\sup |\mu(t)|=\|\mu\|$, and choose a constant $t \in I_{\chi}^{T}$

$$
R \geq \Phi
$$

In view of Lemma 2.8, we define the operators $\mathscr{A}_{1}$ and $\mathscr{A}_{2}$ on the ball $B_{R}=\left\{x \in \mathscr{C}:\|x\|_{\mathscr{C}} \leq R\right\}$ by

$$
\begin{aligned}
\left(\mathscr{A}_{1} x\right)(t) & :=-t^{\beta-1} I_{r}^{\gamma} \Omega_{1}(T) I_{p}^{\beta} I_{q}^{\alpha} f\left(u, x, D_{\theta}^{\mu} x, \Psi_{\omega^{x}}^{v} x\right)(\eta) /(1+\rho(t)) \eta^{\beta-1} \\
& \times\left(I_{r}^{\gamma} \Omega_{1}(T)-\eta^{\alpha} I_{r}^{\gamma} \Omega_{2}(T)\right)
\end{aligned}
$$




$$
\begin{aligned}
\left(\mathscr{A}_{2} x\right)(t) & :=(1+\rho(t))^{-1} I_{p}^{\beta} I_{q}^{\alpha} f\left(u, x, D_{\theta}^{\mu} x, \Psi_{\omega}^{v} x\right)(t)+\left[(\eta t)^{\beta-1}\left(t^{\alpha}-\eta^{\alpha}\right)\right. \\
& \times\left(g(x)-I_{r}^{\gamma}\left((1+\rho(s))^{-1} I_{p}^{\beta} I_{q}^{\alpha} f\left(v, x, D_{\theta}^{\mu} x, \Psi_{\omega}^{v} x\right)\right)(T)\right) \\
& \left.+t^{\alpha+\beta-1} I_{r}^{\gamma} \Omega_{2}(T) I_{p}^{\beta} I_{q}^{\alpha} f\left(u, x, D_{\theta}^{\mu} x, \Psi_{\omega}^{v} x\right)(\eta)\right] / \\
& (1+\rho(t)) \eta^{\beta-1}\left(I_{r}^{\gamma} \Omega_{1}(T)-\eta^{\alpha} I_{r}^{\gamma} \Omega_{2}(T)\right) .
\end{aligned}
$$

For all $x, y \in B_{R}$, we have

$$
\begin{aligned}
& \left|\mathscr{A}_{1} x+\mathscr{A}_{2} y\right| \\
\leq & \|\mu\|\left\{\frac{\Gamma_{p}(\alpha+1) T^{\alpha+\beta}}{\Gamma_{q}(\alpha+1) \Gamma_{p}(\alpha+\beta+1)(1+n)}+\frac{\Gamma_{p}(\alpha+1)(1+N) \eta^{\alpha+1} T^{\alpha+\beta-1} \Lambda_{1}}{\Gamma_{q}(\alpha+1) \Gamma_{p}(\alpha+\beta+1)(1+n)^{2} \Lambda_{2}}\right. \\
& \left.+\frac{\Gamma_{p}(\alpha+1) \Gamma_{r}(\beta+\gamma) \Gamma_{r}(\alpha+\beta+1) \Gamma_{r}(\alpha+\beta+\gamma)(1+N)\left(T^{\alpha}-\eta^{\alpha}\right) T^{\alpha+\beta}}{\Gamma_{q}(\alpha+1) \Gamma_{p}(\alpha+\beta+1) \Gamma_{r}(\alpha+\beta+\gamma+1)(1+n)^{2} \Lambda_{2}}\right\} \\
& +\tau\left\{\frac{\Gamma_{r}(\beta+\gamma) \Gamma_{r}(\alpha+\beta+\gamma)(1+N)\left(T^{\alpha}-\eta^{\alpha}\right)}{(1+n) T^{\gamma} \Lambda_{2}}\right\} \\
= & \|\mu\|\left(P_{1}+P_{2}\right)+\frac{\Gamma_{p}(\alpha+\beta+1) \Gamma_{q}(\alpha+1) \Gamma_{r}(\alpha+\beta+\gamma+1)(1+n)}{\Gamma_{p}(\alpha+1) \Gamma_{r}(\alpha+\beta+1) T^{\alpha+\beta+\gamma}} \tau P_{2} \\
= & \Phi \leq R .
\end{aligned}
$$

By proceeding in a similar way as above and Theorem 3.1, we obtain $\left\|D_{\theta}^{\mu} \mathscr{A}_{1} x+D_{\theta}^{\mu} \mathscr{A}_{2} y\right\|<R$. Hence $\left\|\mathscr{A}_{1} x+\mathscr{A}_{2} y\right\|_{\mathscr{C}}<R$. Therefore, $\mathscr{A}_{1} x+\mathscr{A}_{2} y \in B_{R}$. The condition (3.2) implies that $\mathscr{A}_{2}$ is a contraction mapping.

We next show that $\mathscr{A}_{1}$ is compact and continuous. Continuity of $f$ coupled with the assumption $\left(H_{4}\right)$ implies that the operator $\mathscr{A}_{1}$ is continuous and uniformly bounded on $B_{R}$. For $t_{1}, t_{2} \in I_{\chi}^{T}$ with $t_{1}<t_{2}$, we have

$$
\begin{aligned}
& \left|\mathscr{A}_{1} x\left(t_{2}\right)-\mathscr{A}_{1} x\left(t_{1}\right)\right| \\
\leq & \left|t_{2}^{\beta-1}-t_{1}^{\beta-1}\right| I_{r}^{\gamma} \Omega_{1}(T) I_{p}^{\beta} I_{q}^{\alpha}\left|f\left(u, x, D_{\theta}^{\mu} x, \Psi_{\omega}^{v} x\right)\right|(\eta) / \\
& (1+n) \eta^{\beta-1}\left|I_{r}^{\gamma} \Omega_{1}(T)-\eta^{\alpha} I_{r}^{\gamma} \Omega_{2}(T)\right| \\
\leq & \left|t_{2}^{\beta-1}-t_{1}^{\beta-1}\right|\|\mu\|\left\{\frac{\Gamma_{p}(\alpha+1) \Gamma_{r}(\alpha+\beta) \Gamma_{r}(\beta+\gamma)(1+N) \eta^{\alpha+1} T^{\alpha}}{\Gamma_{q}(\alpha+1) \Gamma_{p}(\alpha+\beta+1)(1+n)^{2} \Lambda_{2}}\right\} .
\end{aligned}
$$


Similarly to the above proof and Theorem 3.1, we obtain

$$
\begin{aligned}
& \left|D_{\theta}^{\mu} \mathscr{A}_{1} x\left(t_{2}\right)-D_{\theta}^{\mu} \mathscr{A}_{1} x\left(t_{1}\right)\right| \\
< & \left|\mathscr{A}_{1} x\left(t_{2}\right)-\mathscr{A}_{1} x\left(t_{1}\right)\right| \\
\leq & \left|t_{2}^{\beta-1}-t_{1}^{\beta-1}\right|\|\mu\|\left\{\frac{\Gamma_{p}(\alpha+1) \Gamma_{r}(\alpha+\beta) \Gamma_{r}(\beta+\gamma)(1+N) \eta^{\alpha+1} T^{\alpha}}{\Gamma_{q}(\alpha+1) \Gamma_{p}(\alpha+\beta+1)(1+n)^{2} \Lambda_{2}}\right\} .
\end{aligned}
$$

We note that when $\left|t_{2}-t_{1}\right| \rightarrow 0$, the right-hand side of the above inequality tends to be zero. So $\mathscr{A}_{1}$ is relatively compact on $B_{R}$. Hence, by the Arzela-Ascoli Theorem, $\mathscr{A}_{1}$ is compact on $B_{R}$.

Therefore, all the assumptions of Theorem 3.2 are satisfied and the conclusion of Theorem 3.2 implies that boundary value problem (1.7) has at least one solution on $I_{\chi}^{T}$. This completes the proof.

\section{Example}

The following example is given to illustrate our main results.

Consider the following fractional $q$-integrodifference boundary value problem

$$
\left\{\begin{array}{l}
D_{\frac{1}{2}}^{\frac{3}{4}}\left(D_{\frac{2}{3}}^{\frac{1}{2}}\left(1+e^{\sin (2 \pi t)}\right)\right) x(t)=\frac{e^{\sin ^{2}(2 \pi t)}}{100+e^{\cos ^{2}(2 \pi t)}} \cdot \frac{|x(t)|+\left|D_{\frac{1}{4}}^{\frac{2}{3}} x(t)\right|+\left|\Psi_{\frac{1}{3}}^{\frac{7}{4}} x(t)\right|}{1+|x(t)|}, t \in I_{\chi}^{1}, \\
x(0)=x\left(\frac{1}{4}\right), \quad I_{\frac{1}{4}}^{\frac{5}{3}}, x(T)=\sum_{i=1}^{n} C_{i} x\left(t_{i}\right),
\end{array}\right.
$$

where $0<t_{1}, t_{2}, \ldots, t_{n}<1$ and $C_{i}$ are given positive constants with $\sum_{i=1}^{n} C_{i}<\frac{1}{100}, \alpha=\frac{3}{4}, \beta=\frac{1}{2}$, $\mu=\frac{2}{3}, v=\frac{7}{4}, \gamma=\frac{5}{3}, \eta=\frac{1}{4}, q=\frac{1}{2}, p=\frac{2}{3}, r=\frac{1}{4}, \theta=\frac{1}{4}, \omega=\frac{1}{3}, T=1, \rho(t)=e^{\sin (2 \pi t)}$,

$$
\begin{aligned}
f\left(t, x, D_{\frac{1}{4}}^{\frac{2}{3}} x, \Psi_{\frac{1}{3}}^{\frac{7}{4}} x\right) & =\frac{e^{\sin ^{2}(2 \pi t)}}{100+e^{\cos ^{2}(2 \pi t)}} \cdot \frac{|x(t)|+\left|D_{\frac{1}{4}}^{\frac{2}{3}} x(t)\right|+\left|\Psi_{\frac{1}{3}}^{\frac{7}{4}} x(t)\right|}{1+|x(t)|}, \\
\Psi_{\frac{1}{3}}^{\frac{7}{4}} x(t) & =\frac{1}{\Gamma_{\frac{1}{3}}\left(\frac{7}{4}\right)} \int_{0}^{t}\left(t-\frac{s}{3}\right)^{\left(\frac{3}{4}\right)} \frac{e^{-(s-t)}}{12} x(s) d_{\omega} s
\end{aligned}
$$

and $\varphi(t, s)=\frac{e^{-(s-t)}}{12}$. Note that

$$
\begin{aligned}
& \left|f\left(t, x, D_{\frac{1}{4}}^{\frac{2}{3}} x, \Psi_{\frac{1}{3}}^{\frac{7}{4}} x\right)-f\left(t, y, D_{\frac{1}{4}}^{\frac{2}{3}} y, \Psi_{\frac{1}{3}}^{\frac{7}{4}} y\right)\right| \\
& \leq \frac{1}{101}|x-y|+\frac{1}{101}\left|D_{\frac{1}{4}}^{\frac{2}{3}} x-D_{\frac{1}{4}}^{\frac{2}{3}} y\right|+\frac{1}{101}\left|\Psi_{\frac{1}{3}}^{\frac{7}{4}} x-\Psi_{\frac{1}{3}}^{\frac{7}{4}} y\right|
\end{aligned}
$$


and $\varphi_{0}=\frac{e}{12},\left(H_{1}\right)$ is satisfied with $L_{1}=L_{2}=L_{3}=\frac{1}{101}$. So,

$$
\lambda=L_{1}+L_{2}+L_{3} \frac{e}{12 \Gamma_{\frac{1}{3}}\left(\frac{11}{4}\right)} \approx 0.0216
$$

We also have

$$
|g(x)-g(y)|=\left|\sum_{i=1}^{n} C_{i} x\left(t_{i}\right)-\sum_{i=1}^{n} C_{i} y\left(t_{i}\right)\right| \leq \sum_{i=1}^{n} C_{i}|x-y| .
$$

So, $\left(H_{2}\right)$ holds with $\tau=\sum_{i=1}^{n} C_{i}<\frac{1}{100}$. Since $\frac{1}{e}<\rho(t)<e$, then $\left(H_{3}\right)$ is satisfied with $N=e$, $n=\frac{1}{e}$. We can show that

$$
\begin{aligned}
& \Lambda_{1}=\left|\Gamma_{\frac{1}{4}}\left(\frac{1}{2}\right) \Gamma_{\frac{1}{4}}\left(\frac{35}{12}\right)-\Gamma_{\frac{1}{4}}\left(\frac{5}{4}\right) \Gamma_{\frac{1}{4}}\left(\frac{13}{6}\right)\right| \approx 0.3348 \\
& \Lambda_{2}=\left|\Gamma_{\frac{1}{4}}\left(\frac{5}{4}\right) \Gamma_{\frac{1}{4}}\left(\frac{13}{6}\right)-\Gamma_{\frac{1}{4}}\left(\frac{1}{2}\right) \Gamma_{\frac{1}{4}}\left(\frac{35}{12}\right)\left(\frac{1}{4}\right)^{\frac{3}{4}}\right| \approx 0.1630, \\
& P_{1}=\frac{\Gamma_{\frac{2}{3}}\left(\frac{7}{4}\right)}{\Gamma_{\frac{1}{2}}\left(\frac{7}{4}\right) \Gamma_{\frac{2}{3}}\left(\frac{9}{4}\right)\left(1+\frac{1}{e}\right)}+\frac{\Gamma_{\frac{2}{3}}\left(\frac{7}{4}\right)(1+e)\left(\frac{1}{4}\right)^{\frac{7}{4}} \Lambda_{1}}{\Gamma_{\frac{1}{2}}\left(\frac{7}{4}\right) \Gamma_{\frac{2}{3}}\left(\frac{9}{4}\right)\left(1+\frac{1}{e}\right)^{2} \Lambda_{2}} \approx 8.3267, \\
& P_{2}=\frac{\Gamma_{\frac{2}{3}}\left(\frac{7}{4}\right) \Gamma_{\frac{1}{4}}\left(\frac{13}{6}\right) \Gamma_{\frac{1}{4}}\left(\frac{9}{4}\right) \Gamma_{\frac{1}{4}}\left(\frac{35}{12}\right)(1+e)\left(1-\left(\frac{1}{4}\right)^{\frac{3}{4}}\right)}{\Gamma_{\frac{1}{2}}\left(\frac{7}{4}\right) \Gamma_{\frac{2}{3}}\left(\frac{9}{4}\right) \Gamma_{\frac{1}{4}}\left(\frac{47}{12}\right)\left(1+\frac{1}{e}\right)^{2} \Lambda_{2}} \approx 21.9253 .
\end{aligned}
$$

Therefore, we get

$$
\Theta=\lambda\left(P_{1}+P_{2}\right)+\frac{\Gamma_{\frac{1}{2}}\left(\frac{7}{4}\right) \Gamma_{\frac{2}{3}}\left(\frac{9}{4}\right) \Gamma_{\frac{1}{4}}\left(\frac{47}{12}\right)\left(1+\frac{1}{e}\right)}{\Gamma_{\frac{2}{3}}\left(\frac{7}{4}\right) \Gamma_{\frac{1}{4}}\left(\frac{9}{4}\right)} \tau P_{2} \approx 0.6815<1 .
$$

Hence, by Theorem 3.1, this problem has a unique solution on $I_{\chi}^{1}$.

\section{Acknowledgment}

The authors are grateful to the reviewers for useful suggestions which improve the contents of this paper.

\section{REFERENCES}

[1] K. Miller, B. Ross, An Introduction to the Fractional Calculus and Fractional Differential Equations, A WileyInterscience Publication, New York, 1993.

[2] K. Oldham, J. Spanier, The Fractional Calculus, Academic Press, New York, 1974.

[3] R. Magin, Fractional Calculus in Bioengineering, Begell House, Redding, 2006.

[4] Y. Zhao, S. Sun, Z. Han, Q. Li, The existence of multiple positive solutions for boundary value problems of nonlinear fractional differential equations, Commun. Nonlinear Sci. Numer. Simul. 16(4) (2011), 2086-2097. 
[5] Y. Zhao, S. Sun, Z. Han, M. Zhang, Positive solutions for boundary value problems of nonlinear fractional differential equations, Appl. Math. Comput. 217 (2011), 6950-6958.

[6] W. Feng, S. Sun, Z. Han, Y. Zhao, Existence of solutions for a singular system of nonlinear fractional differential equations, Comput. Math. Appl. 62(3) (2011), 1370-1378.

[7] C. Goodrich, On discrete sequential fractional boundary value problems, J. Math. Anal. Appl. 385(1) (2012), 111-124.

[8] F. Atici, S. Sengl, Modeling with fractional difference equations, J. Math. Anal. Appl. 369 (2010), 1-9.

[9] W. Yang, Positive solution for fractional $q$-difference boundary value problems with $\phi$-Laplacian operator, Bull. Malays. Math. Sci. Soc. 36(4) (2013), 11951203.

[10] Y. Zhao, H. Chen, Q. Zhang, Existence results for fractional $q$-difference equations with nonlocal $q$-integral boundary conditions, Adv. Differ. Equ. 2013 (2013), Article ID 48.

[11] N. Pongarm, S. Asawasamrit, J. Tariboon, S.K. Ntouyas, Multi-strip fractional $q$-integral boundary value problems for nonlinear fractional q-difference equations, Adv. Diff. Equ. 2014 (2014), Article ID 193.

[12] T. Sitthiwirattham, On a fractional $q$-integral boundary value problems for fractional $q$-difference equations and fractional $q$-intergodifference equations involving different numbers of order $q$, Bound. Value Probl. 2016 (2016), Article ID 12.

[13] N. Patanarapeelert, U. Sriphanomwan, T. Sitthiwirattham, On a class of sequential fractional $q$ integrodifference boundary value problems involving different numbers of $q$ in derivatives and integrals, Adv. Diff. Equ. 2016 (2016), Article ID 148.

[14] R. Ferreira, Nontrivial solutions for fractional $q$-difference boundary value problems, Electron. J. Qual. Theory Differ. Equ. 2010 (2010), Article ID 70.

[15] R.P. Agarwal, Certain fractional $q$-integrals and $q$-derivatives, Proc. Cambridge Philos. Soc. 66 (1969), 365370.

[16] P. Rajkovic, S. Marinkovic, M. Stankovic, Fractional integrals and derivatives in $q$-calculus, Appl. Anal. Discrete Math. 1 (2007), 311-323.

[17] V. Kac, P. Cheung, Quatum calculus, Springer, Newyork, 2000.

[18] A. Granas, J. Dugundji, Fixed point theory, Springer, New York, 2005. 